\title{
Constraining fission parameters for highly excited compound nuclei
}

\author{
Davide Mancusi* and Joseph Cugnon \\ University of Liège, AGO Department \\ allée du 6 août 17, bât. B5 \\ B-4000 Liège 1, Belgium \\ *E-mail:d.mancusi@ulg.ac.be \\ Robert J. Charity \\ Department of Chemistry, Washington University \\ St. Louis, Missouri 63130, USA
}

\begin{abstract}
We present a statistical-model description of fission, in the framework of compoundnucleus decay, which is found to simultaneously reproduce data from both heavy-ioninduced fusion reactions and proton-induced spallation reactions around $1 \mathrm{GeV}$. For the spallation reactions, the initial compound-nucleus population is predicted by the Liège intranucleon cascade model. We are able to reproduce experimental fission probabilities in the all reactions with the same parameter set. We also discuss the need for fission transients, which are expected to have a significant effect on the spallation reactions.
\end{abstract}

Keywords: fusion reactions, spallation reactions, fission transients

\section{Introduction}

Although seventy years have passed since the seminal works of Bohr and Wheeler ${ }^{1}$ and Weisskopf and Ewing ${ }^{2}$ and the establishment of a qualitative understanding of the de-excitation mechanism of excited nuclei, quantitatively accurate and universally applicable models do not exist yet. One way to lift the degeneracy of the ingredients of the model is to explore diverse regions of the compoundnucleus parameter space. The production of excited compound nuclei can proceed from several entrance reactions. There has been a long history of compoundnucleus studies using heavy-ion-induced fusion reactions. These reactions allow one to specify the compound nucleus mass, charge and excitation energy, however a distribution of compound nucleus spins is obtained. Alternatively, one can consider the production of excited compound nuclei through spallation reactions. For these reactions, the need for a model to predict the initial compound-nucleus mass, charge, excitation, and spin distributions adds some uncertainty in our abil- 
ity to constrain the statistical-model parameters by fitting data. However, spallation reactions allow us to explore different regions of compound-nucleus spin and excitation energy than can be probed with fusion reactions alone and thus can be important in parameter fitting.

This paper discusses the application of the GEMINI++ de-excitation model ${ }^{3}$ to the description of fission in fusion and spallation reactions. In the latter case, the description of the entrance channel is provided by a coupling to the Liège Intranuclear Cascade model (INCL). ${ }^{4}$ Both INCL and GEMINI++ are among the most sophisticated models in their own fields. We compare the predictions of the models with experimental residue yields in spallation studies and to fission and evaporation-residue excitation functions measured in heavy-ion induced fusion reactions.

\section{The GEMINI++ and INCL4. 5 models}

GEMINI++ is an improved version of the GEMINI model, developed by R.J. Charity. ${ }^{5}$ The de-excitation of the remnant proceeds through a sequence of binary decays until particle emission becomes energetically forbidden or improbable due to competition with gamma-ray emission. For fissile systems, the total fission yield is obtained from the Bohr-Wheeler formalism ${ }^{1}$ and it competes against emission of nucleons and light nuclei. The width of the mass distribution is taken from systematics compiled by Rusanov et al. ${ }^{9}$

Level densities were calculated with the Fermi-gas form. The level-density parameter $a(U)$ is excitation-energy dependent with an initial fast dependence due to the washing out of shell effects following Ref. 10 and a slower dependence needed to fit the evaporation spectra. The shell-smoothed level-density parameter was assumed to have the form

$$
\widetilde{a}(U)=\frac{A}{k_{\infty}-\left(k_{\infty}-k_{0}\right) \exp \left(-\frac{\kappa}{k_{\infty}-k_{0}} \frac{U}{A}\right)}
$$

which varies from $A / k_{0}$ at low excitation energies to $A / k_{\infty}$ at large values. Here $k_{0}=7.3 \mathrm{MeV}$, consistent with neutron-resonance counting data at excitationenergies near the neutron separation energy, and $k_{\infty}=12 \mathrm{MeV}$. The parameter $\kappa$ defines the rate of change of $\widetilde{a}$ with energy and it is essentially zero for light nuclei (i.e. a constant $\widetilde{a}$ value) and increases roughly exponentially with $A$ for heavier nuclei.

The INCL model ${ }^{4}$ can be applied to collisions between nuclei and pions, nucleons or light nuclei of energy lower than a few GeV. The particle-nucleus collision is modelled as a sequence of binary collisions among the particles present in the system; particles that are unstable over the time scale of the collision, notably $\Delta$ 
resonances, are allowed to decay. The nucleus is represented by a square potential well whose radius depends on the nucleon momentum; thus, nucleons move on straight lines until they undergo a collision with another nucleon or until they reach the surface, where they escape if their total energy is positive and they manage to penetrate the Coulomb barrier.

\section{Adjustment of fission yields}

Fusion and spallation reactions populate different regions of the compoundnucleus excitation-energy/spin plane. For the spallation reaction, the INCL4.5 model predicts average values of about $167 \mathrm{MeV}$ and $16.5 \hbar$, respectively, but both distributions are quite broad and extend up to $\sim 650 \mathrm{MeV}$ and $\sim 50 \hbar$. On the other hand the fusion reactions we considered are characterized by higher spins and lower excitation energies. If we restrict ourselves to the regime of complete fusion $\left(E_{\text {beam }} / A<10 \mathrm{MeV}\right)$, then we can explore somewhat higher excitation energies with more symmetric entrance channels, but high spins will still be populated. Thus it is clear that the comparison between spallation and fusion data represents a promising tool to extend the predictive power of the model over a wide region of mass, energy and spin.

For spallation reactions, we focused our efforts on proton-induced fission reactions on ${ }^{197} \mathrm{Au},{ }^{208} \mathrm{~Pb}$ and ${ }^{238} \mathrm{U}$ around $1 \mathrm{GeV}$, measured in reverse kinematics with the FRagment Separator (FRS) at SIS, GSI, Darmstadt, Germany. ${ }^{12-14,27,28}$

\subsection{Modifications of the fission width}

The Bohr-Wheeler fission width,

$$
\Gamma_{\mathrm{BW}}=\frac{1}{2 \pi \rho_{\mathrm{n}}\left(E^{*}, J\right)} \int \mathrm{d} \epsilon \rho_{\mathrm{f}}\left(E^{*}-B(J)-\epsilon, J\right),
$$

is sensitive to the choice of the fission barrier $B$ and to the level-density parameters $a_{\mathrm{f}}$ and $a_{\mathrm{n}}$ associated with the saddle-point and ground-state configurations. The energy dependence of $a_{\mathrm{f}}$ was assumed identical for ground state and saddle point (Eq. (1)) and the magnitude of the two parameters was varied by a constant scaling factor $a_{\mathrm{f}} / a_{\mathrm{n}}$ to account for the increased surface area of the saddle-point configuration. ${ }^{15}$ Moreover, Lestone ${ }^{16}$ developed a treatment of fission which explicitly included the tilting collective degree of freedom at saddle point. We have tried to reproduce simultaneously fission cross sections from fusion and spallation experiments by (a) scaling the Sierk fission barrier by a constant factor, (b) scaling the decay width by a constant factor, (c) adjusting the $a_{\mathrm{f}} / a_{\mathrm{n}}$ ratio, (d) using either the Bohr-Wheeler or the Lestone formalism and (e) introducing a constant, step-like fission delay. 

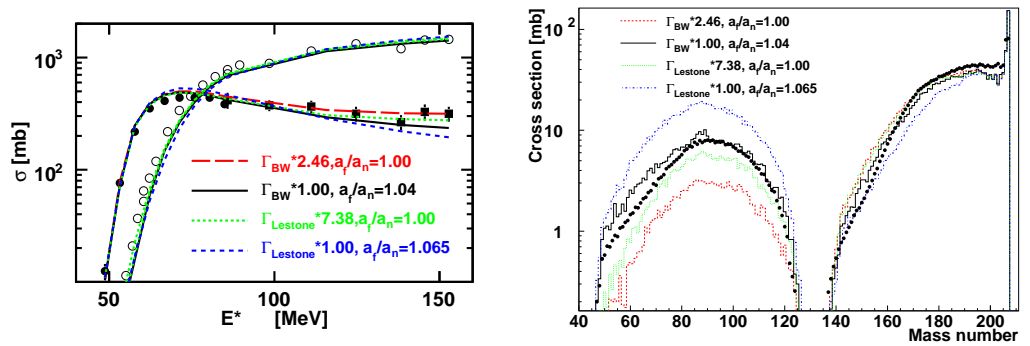

Figure 1. (Color online) Comparison of GEMINI++ predictions to the experimental evaporationresidue and fission excitation fuctions for the ${ }^{19} \mathrm{~F}+{ }^{181} \mathrm{Ta}$ reaction (left) and to experimental residual mass distributions the for the $1-\mathrm{GeV} p+{ }^{208} \mathrm{~Pb}$ reaction (right). Experimental data from Refs. 13,18,19.

\subsection{Results}

Examples of fits to the ${ }^{19} \mathrm{~F}+{ }^{181} \mathrm{Ta} \rightarrow{ }^{200} \mathrm{~Pb}$ fission and evaporation-residue excitation functions are shown in Fig. 1, left panel. As the sum of these quantities (the fusion cross section) is fixed, the degree to which the fission probability is reproduced is best gauged by the fit to the smaller quantity, i.e. the $\sigma_{\text {fis }}$ at low bombarding energies and $\sigma_{\mathrm{ER}}$ at the higher values. Good fits were obtained with four different parameter sets.

As it is impossible to distinguish these four ways of modifying the fission probability from the fusion data alone, we now consider the constraint of adding the spallation data to the analysis. In Fig. 1, right panel, we show the equivalent calculations for the mass distributions of the products produced in the $1-\mathrm{GeV}$ $p+{ }^{208} \mathrm{~Pb}$ spallation reactions. Of all these possibilities, the $\Gamma_{\mathrm{BW}} \times 1.00, a_{\mathrm{f}} / a_{\mathrm{n}}=$ 1.04 calculation reproduces the yield of the fission peak best.

Comparison of GEMINI++ prediction to experimental fission and evaporationresidue excitations functions are shown in Fig. 2. For spallation, Fig. 3 shows the comparison between measured and calculated residue mass distributions. The central result is that it is possible to reproduce the total fission cross section for all the studied spallation reactions with only one free parameter, namely the $a_{\mathrm{f}} / a_{\mathrm{n}}$ ratio.

The solution found is however unique only as long as fission delays are not considered. To show the sensitivity of predictions to these transients, we have incorporated a simple implementation of these in GEMINI++; the fission width is set to zero for a time $t_{\text {delay }}$, after which it assumes the Bohr-Wheeler value. During this fission-delay period, the compound nucleus can decay by light-particle evaporation and intermediate-mass-fragment emission. As Fig. 4 shows, some fission delay can be accommodated for the Lestone fission width with $a_{\mathrm{f}} / a_{\mathrm{n}}=1.065$. 

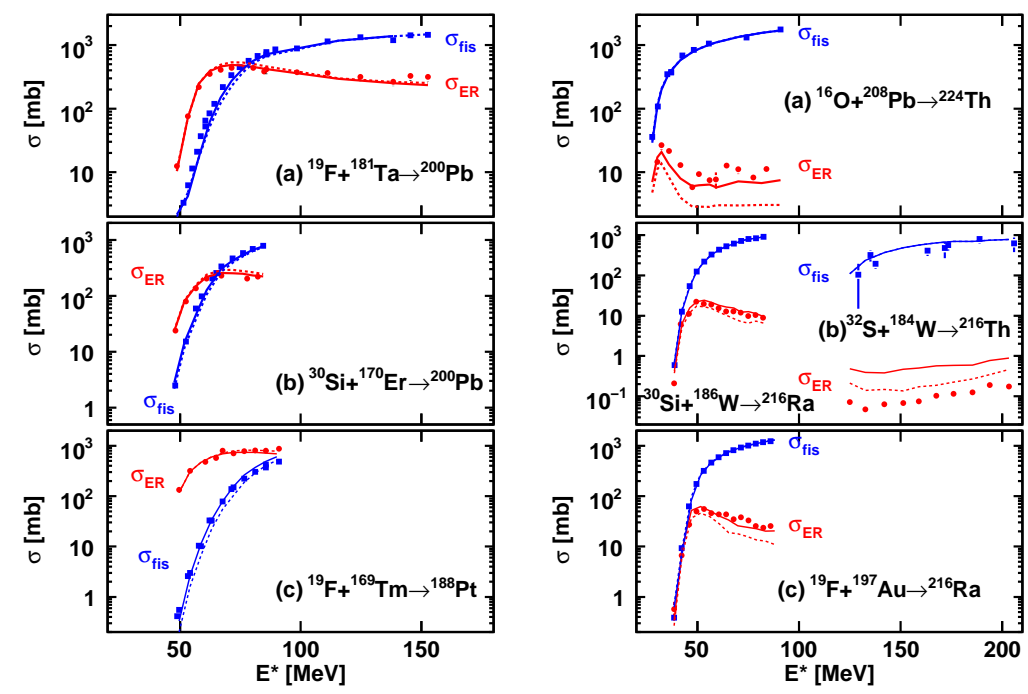

Figure 2. (Color online) Comparison of experimental and calculated fission and evaporation residue excitation function for the indicated reactions. Solid lines: Bohr-Wheeler fission width, $a_{\mathrm{f}} / a_{\mathrm{n}}=1.04$, no fission delay. Dashed lines: Lestone fission width, $a_{\mathrm{f}} / a_{\mathrm{n}}=1.065$, 1-zs fission delay. Experimental data from Refs. 18-26
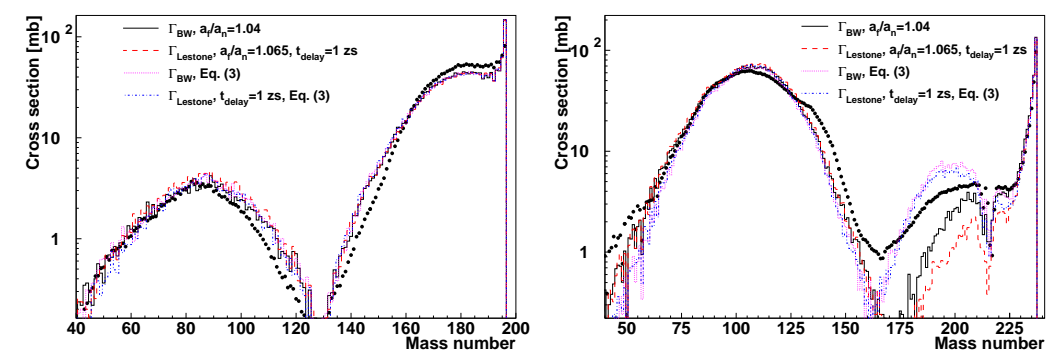

Figure 3. (Color online) Residue-mass distribution for the $p+{ }^{197} \mathrm{Au}$ (left) and $p+{ }^{238} \mathrm{U}$ (right) reactions at $1 \mathrm{GeV}$. Predictions of the INCL4 . 5-GEMINI++ code are shown for different adjustments of the fission width. Experimental data from Ref. 12,14,27,28.

A 1-zs fission delay approximately fits the experimental fission cross section for $1-\mathrm{GeV} p+{ }^{208} \mathrm{~Pb}$. Figs. 2 and 3 also demonstrate that the agreement of the Lestone parameter set, with a constant fission delay of $1 \mathrm{zs}$, is as good as the Bohr-Wheeler parameter set, without any fission delay. 


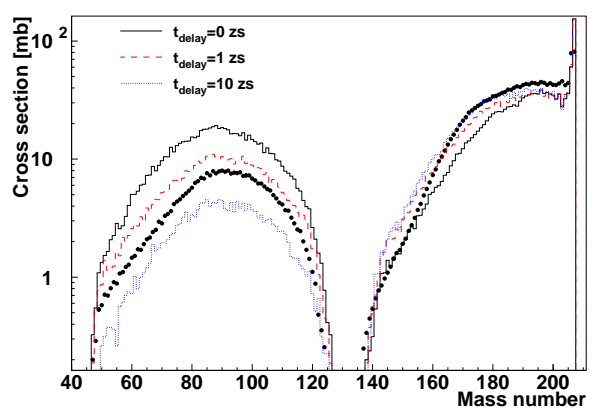

Figure 4. (Color online) Residue-mass distribution for the $p+{ }^{208} \mathrm{~Pb}$ reaction at $1 \mathrm{GeV}$. Predictions of the INCL4 . 5-GEMINI++ code are shown for different values of the fission delay. Experimental data from Refs. $13,18,19$.

\section{Conclusions}

We have described the first coupling of the Liège Intranuclear Cascade model with the GEMINI++ compound-nucleus de-excitation model. We have demonstrated that it is possible to describe fission cross sections from spallation and heavyion fusion reactions within the same framework. The simultaneous application of the statistical-decay model to spallation and fusion actually allows to lift some of the degeneracy of the model parameters. We were able to reproduce all the data with two different parameter sets: one that includes a small fission delay (1 zs) and one that does not. However, the existence of larger fission delays cannot be excluded. Both parameters sets are estimated to be effective for spins up to $60 \hbar$ and excitation energies up to $\sim 300 \mathrm{MeV}$.

\section{Bibliography}

1. N. Bohr and J. A. Wheeler, Phys. Rev. 56, 426(September 1939).

2. V. F. Weisskopf and D. H. Ewing, Phys. Rev. 57, 472(March 1940).

3. R. J. Charity, GEMINI: a code to simulate the decay od a compound nucleus by a series of binary decays, in Joint ICTP-IAEA Advanced Workshop on Model Codes for Spallation Reactions, (Trieste, Italy, 2008). Report INDC(NDC)-0530.

4. A. Boudard, J. Cugnon, S. Leray and C. Volant, Phys. Rev. C 66, p. 044615(October 2002).

5. R. J. Charity, M. A. McMahan, G. J. Wozniak, R. J. McDonald, L. G. Moretto, D. G. Sarantites, L. G. Sobotka, G. Guarino, A. Pantaleo, L. Fiore, A. Gobbi and K. D. Hildenbrand, Nucl. Phys. A 483, 371(June 1988).

6. W. Hauser and H. Feshbach, Phys. Rev. 87, 366(July 1952).

7. R. J. Charity, Systematics of statistical-model parameters in heavy-ion-induced reactions, (2010).

8. L. G. Moretto, Nucl. Phys. A 247, 211(August 1975). 
9. A. Y. Rusanov, M. G. Itkis and V. N. Okolovich, Phys. Atom. Nucl. 60, 683(May 1997).

10. A. V. Ignatyuk, G. N. Smirenkin and A. S. Tishin, Sov. J. Nucl. Phys. 21, p. 255 (1975).

11. J. Cugnon, A. Boudard, S. Leray and D. Mancusi. in preparation, (2009).

12. J. Benlliure, P. Armbruster, M. Bernas, A. Boudard, J. P. Dufour, T. Enqvist, R. Legrain, S. Leray, B. Mustapha, F. Rejmund, K.-H. Schmidt, C. Stéphan, L. TassanGot and C. Volant, Nucl. Phys. A 683, 513(February 2001).

13. T. Enqvist, W. Wlazło, P. Armbruster, J. Benlliure, M. Bernas, A. Boudard, S. Czajkowski, R. Legrain, S. Leray, B. Mustapha, M. Pravikoff, F. Rejmund, K.-H. Schmidt, C. Stéphan, J. Taïeb, L. Tassan-Got and C. Volant, Nucl. Phys. A 686, 481(April 2001).

14. M. Bernas, P. Armbruster, J. Benlliure, A. Boudard, E. Casarejos, S. Czajkowski, T. Enqvist, R. Legrain, S. Leray, B. Mustapha, P. Napolitani, J. Pereira, F. Rejmund, M. V. Ricciardi, K.-H. Schmidt, C. Stéphan, J. Taïeb, L. Tassan-Got and C. Volant, Nucl. Phys. A 725, 213(September 2003).

15. J. Tõke and W. Światecki, Nucl. Phys. A 372, p. 141 (1981).

16. J. P. Lestone, Phys. Rev. C 59, 1540(March 1999).

17. A. J. Sierk, Phys. Rev. C 33, 2039(Jun 1986).

18. D. J. Hinde, J. R. Leigh, J. O. Newton, W. Galster and S. Sie, Nucl. Phys. A 385, p. 109 (1982).

19. A. L. Caraley, B. P. Henry, J. P. Lestone and R. Vandenbosch, Phys. Rev. C 62, p. 054612(Oct 2000).

20. R. J. Charity, L. G. Sobotka, J. F. Dempsey, M. Devlin, S. Komarov, D. G. Sarantites, A. L. Caraley, R. T. deSouza, W. Loveland, D. Peterson, B. B. Back, C. N. Davids and D. Seweryniak, Phys. Rev. C 67, p. 044611(Apr 2003).

21. K.-T. Brinkmann, A. L. Caraley, B. J. Fineman, N. Gan, J. Velkovska and R. L. McGrath, Phys. Rev. C 50, 309(Jul 1994).

22. F. Videbæk, R. B. Goldstein, L. Grodzins, S. G. Steadman, T. A. Belote and J. D. Garrett, Phys. Rev. C 15, 954(Mar 1977).

23. B. B. Back, R. R. Betts, J. E. Gindler, B. D. Wilkins, S. Saini, M. B. Tsang, C. K. Gelbke, W. G. Lynch, M. A. McMahan and P. A. Baisden, Phys. Rev. C 32, 195(July 1985).

24. B. B. Back, D. J. Blumenthal, C. N. Davids, D. J. Henderson, R. Hermann, D. J. Hofman, C. L. Jiang, H. T. Penttilä and A. H. Wuosmaa, Phys. Rev. C 60, p. 044602(August 1999).

25. J. G. Keller, B. B. Back, B. G. Glagola, D. Henderson, S. B. Kaufman, S. J. Sanders, R. H. Siemssen, F. Videbaek, B. D. Wilkins and A. Worsham, Phys. Rev. C 36, 1364(Oct 1987).

26. A. C. Berriman, D. J. Hinde, M. Dasgupt, C. R. Morton, R. D. Butt and J. O. Newton, Nature (London) 413, p. 144 (2001).

27. J. Taïeb, K.-H. Schmidt, L. Tassan-Got, P. Armbruster, J. Benlliure, M. Bernas, A. Boudard, E. Casarejos, S. Czajkowski, T. Enqvist, R. Legrain, S. Leray, B. Mustapha, M. Pravikoff, F. Rejmund, C. Stéphan, C. Volant and W. Wlazło, Nucl. Phys. A 724, 413(September 2003).

28. M. V. Ricciardi, P. Armbruster, J. Benlliure, M. Bernas, A. Boudard, S. Czajkowski, T. Enqvist, A. Kelić, S. Leray, R. Legrain, B. Mustapha, J. Pereira, F. Rejmund, K.-H. Schmidt, C. Stéphan, L. Tassan-Got, C. Volant and O. Yordanov, Phys. Rev. C 73, p. 014607(January 2006). 